\title{
Serosurveillance of Anti SARS-Cov-2 Antibodies among Essential Workers in Navi Mumbai - A Single Centre Study
}

\author{
Ujwala Maheshwari $^{1}$, Jyotsna Sahai ${ }^{2}$, Vivek Hebbar ${ }^{3}$ \\ ${ }^{1}$ Professor, ${ }^{2}$ Senior Resident, ${ }^{3}$ Physician, MGM Medical College and Hospital, Kamothe, Navi Mumbai \\ Corresponding Author: Jyotsna Sahai
}

\begin{abstract}
Background - COVID - 19 is the latest raging pandemic that the world is facing at the moment. Frontline workers have been highly exposed to this disease and majority of them have suffered from this deadly disease too. Studying the prevalence of antibodies among frontline workers in different areas is crucial for understanding the potential risk of transmission, the prevalence of herd immunity, vaccine deployment and risk stratification in different areas of work.

Materials and Methods - A prospective study was conducted in a tertiary care hospital of Navi Mumbai on 200 essential service providers, $100 \mathrm{HCWs}$ and 100 police personnel, of which 2 police personnel dropped out of the study. This study was conducted over a period of 2 days, i.e., $20^{\text {th }}$ and $21^{\text {st }}$ May 2021 and it included answering a questionnaire and drawing blood to detect SARS COV-2 antibodies.

Results - Out of the total 198 participants, there were 130 males and 68 females. Most of the participants were in the $30-39$ years age group and $41 \%$ participants had suffered from COVID-19 in the past one year. $60.10 \%$ participants had developed IgG antibodies against COVID-19. The highest titre of antibodies found was between 1-10 amongst both groups followed by antibody levels more than 100 among HCWs. Subsequently, it was found that there was a $72 \%$ seropositivity rate amongst frontline workers whereas only a $48 \%$ positivity rate in HCWs.

Conclusion - Measuring antibody titres allows determination of prevalence of COVID - 19 infection amongst heavily exposed individuals, who have been badly affected by the 2 waves of this pandemic. This serosurvey also helped us confirm that there is a declining trend of antibodies during convalescence phase and that even though the infectivity rate is high amongst essential service providers, not all developed a sustained antibody response. These findings uphold the need for strict precautionary measures and that laxity must not be observed while following the same to contain the pandemic.
\end{abstract}

Keywords: Antibodies, COVID -19, SARS-COV-2, Serosurveillance, Health Care Workers, Police Personnel, Frontline Workers.

\section{INTRODUCTION}

COVID-19 or Corona Virus Disease - 2019 is caused by a virus belonging to SARS genus and named as SARS-CoV-2. What initially started as a cluster outbreak in Wuhan city of China later turned into a pandemic with dreadful consequences worldwide. As of $28^{\text {th }}$ May 2021, India had reported 27,564,857 cases and 319,007 deaths and was $2^{\text {nd }}$ worldwide in terms of caseload. ${ }^{(1)}$ Maharashtra has been the worst affected state in the country in terms of case load and death toll and this can partly be attributed to the fact that COVID - 19 presents with a wide variety of symptoms and signs and is often detected late or is asymptomatic in carriers. This causes 
additional burden on an already stretched health care system.

Surveillance systems form the foundation stone of active case finding, testing and contact tracing, which are the key components of the public health response to this novel, emerging infectious disease. ${ }^{(2)}$

The WHO global research map for COVID-19 and others recommend population-level sero-epidemiological studies to generate data on the levels of infection in populations and recommend containment measures accordingly. ${ }^{(3)}$

Serosurveys involve collection of specimens to measure the presence and level of antigen-specific antibodies in a group of people. It reveals what proportion of the population has been exposed to an infectious disease or has been vaccinated against a pathogen. It also estimates the level of population immunity to one or more infectious diseases, sero surveys identify gaps in immunity because people were not vaccinated or previously infected. Immunity gaps can be in specific age groups, certain locations, or among specific populations such as migrants. Identifying immunity gaps can guide immunization programs. Thus, it estimates parameters for modeling and transmission dynamics to measure disease burden and guide immunization programs. (4)

Studying the prevalence of antibodies among frontline workers in different areas is crucial for understanding the potential risk of transmission, the prevalence of herd immunity, vaccine deployment and risk stratification in different areas of work. ${ }^{(5)}$

Sero surveillance has been done in many different cohorts with prevalence ranging from $2.67 \%$ to $24.4 \%$. $^{(6,7)}$

The last sero surveillance undertaken in March 2021 by the municipal corporation in Mumbai showed a seropositivity rate of $36.3 \%$.

The goal of our study was to estimate the sero prevalence of anti SARS -
COV-2 antibodies among frontline essential workers.

\section{MATERIALS AND METHODS}

A prospective, observational study was designed and conducted at a tertiary care hospital in Navi Mumbai after taking ethical clearance from the institutional ethical committee. 200 participants were enrolled in the survey, with 100 healthcare workers and 100 police officers currently posted in Navi Mumbai area after obtaining their written informed consent. The healthcare workers included all staff in direct or indirect contact with COVID - 19 patients (i.e., Doctors, Nurses, Ward staff, Laboratory Technicians, Pharmacists and Administrative Staff). All participants included in the study were aged between 18 -60 years of age, were not suffering from COVID - 19 at the time of survey or anytime in the last one month from the time of survey and were not pregnant or lactating at the time of survey.

Amongst the 100 police officers, 2 officers dropped out of the study due to one of them testing positive for COVID - 19 (1) and the other being a lactating mother (1). Thus, 98 police officers were finally enrolled for the survey.

The period of study was two days, i.e, $20^{\text {th }}-21^{\text {st }}$ May 2021 and was conducted at MGM hospital, Navi Mumbai. The study procedure included answering a questionnaire and drawing blood to detect SARS CoV-2 antibodies.

All data was collected in a strictly confidential manner according to the study protocol. The questionnaire included information about gender, age, whether participant had suffered from COVID - 19 anytime during the last one year, their vaccination status, which vaccine had they taken and if any comorbidities.

Venous blood samples were collected in a $3 \mathrm{ml}$ gel vacutainer. Antibodies against SARS- CoV-2 in serum were analyzed using electrochemiluminescence immunoassay on Elecsys ${ }^{\circledR}$, Roche, Switzerland. The ECLIA 
test kit is a semi-quantitative in vitro assay for human antibodies against SARS-CoV- 2 structural proteins in serum.

\section{RESULTS}

Out of the total 198 participants enrolled in the survey, there were 130 males and 68 females. Amongst the different age groups of people surveyed, it was found that the maximum number of candidates sampled were in the $30-39$ years age group. (Table 1)

Table 1 - Age group wise distribution of participants.

\begin{tabular}{|l|l|l|l|}
\hline Age Group & Males & Females & Total \\
\hline $20-29$ & 24 & 29 & 53 \\
\hline $30-39$ & 58 & 29 & 87 \\
\hline $40-49$ & 30 & 6 & 36 \\
\hline $50-59$ & 18 & 4 & 22 \\
\hline Total & 130 & 68 & 198 \\
\hline
\end{tabular}

82 participants (41\%) had suffered from COVID-19 infection in the past one year, of which there were 45 males and 37 females. 159 participants $(80 \%)$ had no comorbidities, where as HTN and DM were the most common comorbidities encountered in our study, 20 and 15 participants respectively.

Amongst the 198 participants, there were 183 participants $(92 \%)$ who had taken the vaccine (167 had taken both doses and 16 had taken only the $1^{\text {st }}$ dose of the vaccine). 15 participants (8\%) had not taken any vaccine.

Out of the two vaccines currently approved for administration by the Indian government, it was found that $90 \%$ (165) participants had taken Covishield vaccine and $10 \%$ (18) participants had taken Covaxin doses.

We took the cut off for antibody development as 1 and any person whose $\mathrm{IgG}$ antibody titres were less than 1 was counted as seronegative during our survey. Taking this criterion into consideration, it was seen that 119 persons had developed antibodies against SARS $-\mathrm{CoV}-2$ where as 79 persons had not developed IgG antibodies. (Tables 2 and 3)

Table 2 - Antibody titres amongst both essential service workers divided according to gender.

\begin{tabular}{|l|l|l|l|l|l|}
\hline Antibody Titres & Frontline Workers (police personnel) & Health Care Workers (HCWs) & Total \\
\hline & Males & Females & Males & Females & \\
\hline$>$ or $=1$ & 59 & 15 & 25 & 20 & $119(60.10 \%)$ \\
\hline$<1$ & 21 & 3 & 25 & 30 & $79(39.90 \%)$ \\
\hline Total & 80 & 18 & 50 & 50 & 198 \\
\hline
\end{tabular}

Table 3 - Antibody titres amongst participants with and without Covid - 19 infection.

\begin{tabular}{|l|l|l|l|l|l|}
\hline Antibody Titres & \multicolumn{2}{|l|}{ Frontline Workers } & Health Care Workers & Total \\
\hline & $\begin{array}{l}\text { Suffered from } \\
\text { Covid - 19 }\end{array}$ & Not suffered from Covid - 19 & $\begin{array}{l}\text { Suffered from Covid } \\
\mathbf{1 9}\end{array}$ & $\begin{array}{l}\text { Not suffered } \\
\text { Covid - 19 }\end{array}$ & from \\
\hline$>$ or $=1$ & 27 & 44 & 46 & 2 & 119 \\
\hline$<1$ & 0 & 27 & 9 & 43 & 79 \\
\hline Total & 27 & 61 & 55 & 45 & 198 \\
\hline
\end{tabular}

Table 4 - Range of antibody titres developed among the study population.

\begin{tabular}{|l|l|l|l|}
\hline $\begin{array}{l}\text { Antibody Titre } \\
\text { Ranges }\end{array}$ & $\begin{array}{l}\text { Front Line } \\
\text { workers }\end{array}$ & $\begin{array}{l}\text { Health Care } \\
\text { Workers }\end{array}$ & Total \\
\hline $0-1$ & 24 & 55 & 79 \\
\hline $1-10$ & 34 & 14 & 48 \\
\hline $11-20$ & 8 & 2 & 10 \\
\hline $21-30$ & 6 & 6 & 12 \\
\hline $31-40$ & 5 & 2 & 7 \\
\hline $41-50$ & 6 & 5 & 11 \\
\hline $51-60$ & 4 & 0 & 4 \\
\hline $61-70$ & 0 & 3 & 3 \\
\hline $71-80$ & 2 & 2 & 4 \\
\hline $81-90$ & 3 & 0 & 3 \\
\hline $91-100$ & 0 & 1 & 1 \\
\hline $101-200$ & 6 & 10 & 16 \\
\hline Total & 98 & 100 & 198 \\
\hline
\end{tabular}

In our study we found that the majority of the population had not developed antibodies against SARS COV 2 and amongst those who had developed antibodies, the highest level of titres found was between $1-10$ among both groups followed by antibody levels more than 100 among health care workers and almost an equal distribution among frontline police personnel.

Taking this data into consideration, the sero-reactivity rates amongst the essential workers is presented in table 5.

Table 5 - Seroreactivity rates amongst Essential workers.

\begin{tabular}{|l|l|l|}
\hline \multicolumn{3}{|c|}{ Table 5 - Seroreactivity rates amongst Essential workers. } \\
\hline Seropositivity & Frontline workers & HCWs \\
\hline Seronegativity & $72 \%$ & $48 \%$ \\
\hline
\end{tabular}




\section{DISCUSSION}

COVID-19 pandemic has over burdened our health care system. HCWs and frontline workers are amongst the worst affected and this has led to a very high toll of lives in these two very essential service sectors.

This study aimed to find the levels of SARS- COV - 2 IgG antibodies, among essential service workers in Navi Mumbai. Presence of $\operatorname{IgG}$ antibodies is suggestive of past infection, either symptomatic or asymptomatic. It could have been in asymptomatic or mildly symptomatic people giving a negative history for Covid - 19 infecion.

This study found that the overall sero-positvity rate was higher in males as compared to females amongst frontline workers. In contrast, among $\mathrm{HCWs}$, the numbers were identical. This finding is contradictory to that of Veluswamy et al, who reported a higher sero-positivity rate in females $(35 \%)$ in comparison to males $(30 \%)$ across various cities all over India during their 6 month study. ${ }^{(8)}$

In our study it was found that seropositivity was the highest in the $30-39$ years age group. This was probably due to maximum exposure of this age group and the chances of being multiply exposed too.

Since this survey was conducted on essential service workers, it was seen that $41 \%$ of the participants had suffered from laboratory confirmed COVID - 19 infection in the past one year.

$60 \%$ participants showed seropositivity in our study, which was higher than the percentage of confirmed COVID -19 cases. This suggested that many of the participants had suffered from asymptomatic or clinically undetected COVID - 19 infections.

Variable sero-reactivity has been reported in the literature, ranging from $57 \%$ prevalence in Bergamo-Italy's epicenter, $20 \%$ in New York City, $5.2 \%$ in Kenya, to a low percentage of $4.7 \%$ in Los Angeles County and $2.8 \%$ in Santa Clara County California. (9-13) Overall SARS-CoV-2 seroprevalence of $24.4 \%$ in HCWs was significantly greater than the $6 \%$ seroprevalence in the general population of the Midlands region, according to data published by Public Health England which suggests a marked occupational risk of exposure for HCWs during the COVID-19 pandemic. ${ }^{(14)}$ Another study found $13.7 \%$ IgG prevalence among 40,329 HCWs in the greater New York City area similar to the community prevalence in New York State $(14.0 \%)$. (11) Hains et al reported a seroconversion in $44.0 \% \quad(n=11 / 25)$ of $\mathrm{HCW}$ s at a dialysis unit in America. ${ }^{(15)} \mathrm{A}$ study in Mumbai on $244 \mathrm{HCW}$ s reported that the prevalence of infection in asymptomatic persons was $4.3 \%$ and in previously symptomatic untested HCWs was $70 \%$. (16)

There were 9 healthcare workers who gave history of suffering from COVID -19 but had antibody titres less than 1 . All of them suffered from mild or asymptomatic COVID - 19 and opted for home isolation.

Some initial studies have shown that $40 \%$ of asymptomatic subjects and $12.9 \%$ of symptomatic subjects became seronegative for IgG antibodies in the "early convalescent phase", there are other isolated reports of rapid decay of $\mathrm{IgG}$ antibodies in persons with mild infections. $(17-20)$ The longevity of the immune response and the level of neutralizing antibodies needed for protection remain unclear, as cases of relapse/reinfection with SARS-CoV-2 have been reported. ${ }^{(17)}$

The variation in the immune response could be attributed to the symptomatology, being less in milder infections, the constitution of individuals, or nutritional status. (21) Thus, further studies are needed to better understand the possible relationship between these factors and variation in the development of antibodies. (22)

Table 6 shows majority of the participants had developed antibodies against COVID - 19 infection and that the association between antibody development and infection is significantly strong. 
Ujwala Maheshwari et.al. Serosurveillance of anti SARS-Cov-2 antibodies among essential workers in Navi Mumbai - a single centre study.

Table 6 - Statistical analysis of antibody development amongst covid infected and non infected individuals.

\begin{tabular}{|l|l|l|l|}
\hline & $\begin{array}{l}\text { COVID } \\
\text { Positive }\end{array}$ & $\begin{array}{l}\text { COVID } \\
\text { Negative }\end{array}$ & Total \\
\hline Antibody Positive & 73 & 46 & 119 \\
\hline Antibody Negative & 9 & 70 & 79 \\
\hline Total & 82 & 116 & 198 \\
\hline Chi Square test Statistic 48.8287 & \\
$P$ Value - --- <0.00001, Significant & \\
\hline
\end{tabular}

The small sample size and absence of serial sampling of the same individual are limitations of the study. Larger serosurveys in HCWs and comparing them to the general population will help in further defining the epidemiology of the illness. At the same time, the presence of antibodies in HCWs should not be equated with immunity or allow for the relaxation of infection control guidelines and practice

\section{CONCLUSIONS}

This study was conducted as a onetime survey to study the antibody titers and sero-reactivity rates prevalent in frontline and HCWs, who have been the most exposed during this pandemic. Measuring antibody titres allows determination of prevalence of COVID - 19 infection in densely populated areas or heavily exposed individuals, who have been badly affected by the 2 waves of the pandemic. Many persons have either suffered from mild or asymptomatic COVID - 19 infections, that have not been reported and / or identified / detected and notified.

This serosurvey shows us the mirror about prevalence of infection as well as antibody levels in essential service workers. We also found that a few participants didn't develop antibodies or had a declining trend of antibodies, suggestive of "decaying antibody titres" or waning IgG levels during convalescence phase. These findings suggest that even though infectivity rates are higher amongst essential service workers, not all developed a sustained antibody response post infection. This finding substantiates the need for stringent SMS (Sanitizing, Masking and Social distancing) criteria to be followed and no relaxation be allowed. Following strict precautionary measures, India would be able to save many more lives, of both the general public as well as essential service workers, and avert a preventable $3^{\text {rd }}$ wave of COVID -19 , which is projected to occur very soon.

\section{Acknowledgement: None}

\section{Conflict of Interest: None}

\section{Source of Funding: None}

\section{Ethical Approval: Approved}

\section{REFERENCES}

1. Worldometers.info, Available from https://www.worldometers.info/coronavirus, accessed on May 28, 2021.

2. World Health Organization. Global surveillance for COVID-19 caused by human infection with COVID- 19 virus: Interim guidance, 20 March 2020. Geneva: WHO; 2020.

3. World Health Organization. Coordinated global research roadmap: 2019 novel coronavirus; March 2020. Geneva: WHO; 2020.

4. Kempen J H, Abashawl A, Kinfemichael H, Difabachew M N, Kempen C J, Debele M T, et al. SARSCoV- 2 Serosurvey in Addis Ababa, Ethiopia. medRxiv. 2020.

5. $\mathrm{Qu} \mathrm{J}, \mathrm{Wu} \mathrm{C}, \mathrm{Li} X$, et al. Profile of immunoglobulin $\mathrm{G}$ and $\operatorname{IgM}$ antibodies against severe acute respiratory syndrome coronavirus 2 (SARS-CoV-2). Clin Infect Dis. 2020;71:2255-8.

6. Kumar MS, Bhatnagar T, Manickam P, Kumar VS, Rade K, Shah N et al. National sero-surveillance to monitor the trend of SARS-CoV-2 infection transmission in India: Protocol for community-based surveillance. Indian J Med Res. 2020 May; 151(5):419-23.

7. Brown TS, Walensky RP. Serosurveillance and the COVID-19 Epidemic in the US: Undetected, Uncertain, and Out of Control. JAMA. 2020 Aug 25; 324(8):749-51.

8. Veluswamy A, Nikam C, Suraweera W, Fu $\mathrm{SH}$, Gelband H, Brown P, et al. SARSCOV-2 Seroprevalence in 12 cities of India from July-December 2020. [PREPRINT] medRxiv. 2021. 
9. Uyoga S, Adetifa IMO, Karanja HK, et al. Seroprevalence of anti-SARS-CoV-2 IgG antibodies in Kenyan blood donors [PREPRINT] medRxiv. 2020

10. Berardi C, Antonini M, Genie MG, Cotugno G, Lanteri A, Melia A et al. The COVID-19 pandemic in Italy: Policy and technology impact on health and non-health outcomes. Health Policy Technol. 2020 Dec; 9(4):45487.

11. Brant-Zawadzki M, Fridman D, Robinson PA, et al. SARS-CoV-2 antibody prevalence in health care workers: preliminary report of a single center study. PLoS One. 2020;15:0

12. Bendavid E, Mulaney B, Sood N, et al. COVID-19 antibody seroprevalence in Santa Clara County, California [PREPRINT] medRxiv. 2020

13. Sood N, Simon P, Ebner P, Eichner D, Reynolds J, Bendavid E, et al. Seroprevalence of SARS-CoV-2-specific antibodies among adults in Los Angeles County, California, on April 10-11, 2020. JAMA. 2020;323:2425-7

14. Shields A, Faustini SE, Perez-Toledo M, Jossi S, Aldera E, Allen JD et al. SARSCoV-2 seroprevalence and asymptomatic viral carriage in healthcare workers: a crosssectional study. Thorax. 2020 Dec; 75(12):1089-94

15. Hains DS, Schwaderer AL, Carroll AE, Starr MC, Wilson AC, Amanat F, et al. Asymptomatic Seroconversion of Immunoglobulins to SARS-CoV-2 in a Pediatric Dialysis Unit. JAMA. 2020 Jun 16; 323(23):2424-5.

16. Singhal T, Shah S, Naik R, Kazi A, Thakkar P. Prevalence of COVID-19 antibodies in healthcare workers at the peak of the pandemic in Mumbai, India: A preliminary study. Indian J Med Microbiol. 2020 JulDec; 38(3 \& 4):461-3.

17. Nag DS, Chaudhry R, Mishra M, Rai S, Gupta M. A prospective study on rapidly declining SARS-CoV-2 IgG antibodies within one to three months of testing IgG positive: can it lead to potential reinfections? Cureus. 2020;12:0.

18. Zeng C, Evans JP, Pearson R, et al. Neutralizing antibody against SARSCoV-2 spike in COVID-19 patients, health care workers, and convalescent plasma donors. JCI Insight. 2020;5:0.

19. Ibarrondo FJ, Fulcher JA, Goodman-Meza $\mathrm{D}$, et al. Rapid decay of anti-SARS-CoV-2 antibodies in persons with mild Covid-19. N Engl J Med. 2020;383:1085-7.

20. Lin Q, Zhu L, Ni Z, Meng $\mathrm{H}$, You L. Duration of serum neutralizing antibodies for SARS-CoV- 2: lessons from SARS-CoV infection. J Microbiol Immunol Infect. 2020;53:821-822.

21. Calder PC. Nutrition, immunity and COVID-19. BMJ Nutr Prev Health. 2020; 3:74-92.

22. Mishra M, Chaudhry R, Rana F, Nag DS, Rai S. Serosurveillance of Health Care Workers in a COVID Hospital: Immune Response, and Its Longevity. Cureus 2021;13(3): e14020. https://dx.doi.org/10.7759\%2Fcureus. 14020

How to cite this article: Maheshwari U, Sahai J, Hebbar V. Serosurveillance of anti SARS-Cov-2 antibodies among essential workers in Navi Mumbai - a single centre study. Int J Health Sci Res. 2021; 11(7): 99-104. DOI: https://doi.org/ 10.52403/ijhsr.20210714 